\title{
Cascade genetic testing for mismatch repair gene mutations
}

\author{
R. J. Mitchell · R. K. Ferguson · A. Macdonald • \\ M. G. Dunlop · H. Campbell · M. E. Porteous
}

Published online: 4 April 2008

(C) Springer Science+Business Media B.V. 2008

\begin{abstract}
Mismatch repair gene mutation carriers have a high risk of developing colorectal cancer, and can benefit from appropriate surveillance. A combined population based ascertainment cascade genetic testing approach provides a systematic and potentially effective strategy for identifying such carriers. We have developed a Markov Chain computer model system which simulates various factors influencing cascade genetic testing; including demographics, uptake, genetic epidemiology and family size. This was used to evaluate cascade genetic testing for mismatch repair gene mutations in theory and practice. Simulations focussed on the population of Scotland by way of illustration, and were based on a 20-year programme in which index cases were ascertained from colorectal cancer cases aged $<55$ years at onset. Results indicated that without practical barriers to cascade genetic testing, 545
\end{abstract}

R. J. Mitchell ( $₫) \cdot$ H. Campbell

Public Health Sciences, University of Edinburgh,

Teviot Place, Edinburgh EH89AG, UK

e-mail: R.J.Mitchell@ed.ac.uk

R. J. Mitchell - M. G. Dunlop - H. Campbell · M. E. Porteous Colon Cancer Genetics Group, School of Molecular \& Clinical

Medicine, University of Edinburgh, Edinburgh, UK

R. K. Ferguson

Department of Computer Sciences, Heriot-Watt University, Edinburgh, UK

\section{A. Macdonald}

Department of Actuarial Mathematics and Statistics,

The Maxwell Institute for Mathematical Sciences,

Heriot-Watt University, Edinburgh, UK

M. E. Porteous

SE Scotland Genetic Service Western General Hospital,

Edinburgh, UK
(95\% CI $=522,568)$ carriers could be identified; $42 \%$ of the population total. This comprised approximately 140 index cases, 302 asymptomatic relatives and 104 previously affected relatives. However, when realistic ascertainment and acceptance rates were used to inform simulations, only $257(95 \% \mathrm{CI}=246,268)$ carriers, about $20 \%$ of the carrier population, were identifiable. Of these approximately 112 were index cases, 108 were asymptomatic relatives, and 37 were previously affected relatives. This contrast emphasises the importance of ascertainment and acceptance rates. Likewise the low number of index cases shows that case identification is a limiting factor. In the absence of robust data from epidemiological studies, these findings can inform decisions about the use of cascade genetic testing for mismatch repair gene mutations.

Keywords Colorectal cancer - Genetic screening · Mismatch repair - Genetic services - Genetic epidemiology

$\begin{array}{ll}\text { Abbreviations } \\ \text { MMR } & \text { Mismatch repair } \\ \text { CRC } & \text { Colorectal cancer } \\ \text { CGTM } & \text { Cascade Genetic Testing Model } \\ \text { GPM } & \text { Genetic Population Model }\end{array}$

\section{Introduction}

A small but significant sub-set of colorectal cancer (CRC) cases are caused by pathogenic mutations in mismatch repair (MMR) genes [1-6], which confer an approximate lifetime risk of $80 \%$ in males and $40 \%$ in females [7]. Colonoscopic screening of carriers can have significant health benefits in terms of both reduced incidence and lifeyears saved [8]. Consequently, there is a compelling 
rationale for identifying MMR gene mutation carriers before they develop disease.

This presents a major challenge, however. Population prevalence is uncertain but is certainly low: the carrier frequency in Scotland has been estimated to be approximately $1: 3,139(95 \% \mathrm{CI}=1: 1,247,1: 7,626)$ [9]. By extrapolation, this implies that there are about 1,200 carriers aged 15-74 in the Scottish population of around 5 million. Population genetic testing for MMR gene mutations is not currently viable, and further information regarding the underlying genetic epidemiology and the effectiveness of intervention strategies is needed before it can even be considered [10-14]. Alternative approaches are thus required.

One current strategy for finding asymptomatic mutation carriers is to offer genetic testing to close relatives of a known carrier. The specific mutation present can thus be traced through an expanded pedigree in a process known as cascade genetic testing. In practice, index cases are often selected on the basis of family history. Tests can be offered to first-degree relatives only, or to a wider group of relatives; a concept which can be referred to as "depth" of testing.

Cascade and population screening represent opposite ends of a continuum. A further option for identifying asymptomatic MMR gene mutation carriers, which is potentially both feasible and effective, could, therefore, be to combine features of both methodologies. A systematic, population-based approach to carrier ascertainment would thus be targeted to individuals at a relatively high risk of carrying a mutation, for example those with early onset CRC. Cascade genetic testing would then be employed, using these individuals as starting points. By only testing individuals at very high risk of harbouring a pathogenic mutation, such an approach would limit the number of non-carriers tested whilst maximizing the number of carriers identified.

The hypothesis that a combined population based ascertainment cascade approach may be both effective and efficient is supported by theoretical work [15]. Cascade genetic testing has also been systematically applied to other complex diseases with adult onset, notably familial hypercholesterolaemia [16, 17], for which it has been shown to be cost effective [18, 19]. However, the outcomes of cascade genetic testing are highly context specific, and this strategy remains to be thoroughly evaluated at the population level for MMR gene mutations. The overarching aim of this paper is to address this gap in current knowledge.

\section{Methods}

\section{Overview}

The complex process of cascade genetic testing was evaluated using a computer model system, implemented using
Java and the database software MySQL. Design was function driven, but in applying probabilities to events in a stochastic manner the model system is essentially an example of a Markov Chain.

The model system has the flexibility to be applied to any population. Results presented here used the Scottish population for illustrative purposes. The model system comprises two integrated models, which operate sequentially:

1. Genetic Population Model (GPM)—simulates demographics, family structure and genetic epidemiology in a dynamic population.

2. Cascade Genetic Testing Model (CGTM)-superimposes cascade genetic testing on populations simulated by the GPM.

\section{Genetic Population Model (GPM)}

The GPM constitutes a flexible and powerful research tool for studying the inheritance of pathogenic mutations at the population level. A dynamic population is simulated over time, with demographics and mutation prevalence at the starting point defined by the model user. A set of variables is assigned for each individual: these include a unique identifier, year of birth, sex, mutation status, disease status and date of death. In the course of the simulation these variables are updated annually, using a series of algorithms which apply a probability for the event in question. In this manner, the occurrence of events such as births, death and disease onset over time is simulated. Obtaining input probability values from real data enables actual demographic and epidemiological trends to be simulated. Importantly, the probability of disease is determined by mutation status as well as age, sex and cohort-specific population incidence. Hence information about the prevalence and penetrance of MMR gene mutations was used to simulate their role in CRC.

Another crucial feature is that partnerships are established within the population, and 'birth' algorithms are applied to females with a current partner, with age-specific probability of birth adjusted to account for existing parity. For each birth event, mother and father identity is known and mutation status can be assigned according to Mendelian inheritance. Hence realistic family relationships and inheritance patterns evolve in the simulated population.

To provide the necessary framework for simulating cascade genetic testing beginning in the present day, the GPM was used to produce a dynamic simulation of the Scottish population from the time of the 1901 census to the year 2049 .

In view of the uncertainty surrounding the prevalence and penetrance of MMR gene mutations (see "Discussion"), these input values were considered as assumptions 
rather than robust estimates. A single estimate of $0.016 \%$, extrapolated from the study by Dunlop et al. [9], was used to represent the collective allele frequency for pathogenic mutations in hMLH1 and hMSH2. Penetrance was estimated to be $80 \%$ in males and $40 \%$ in females $[3,7]$.

A summary of key inputs to the GPM is shown in Appendix 1.

\section{Cascade Genetic Testing Model (CGTM)}

The CGTM simulates the progress of a cascade genetic testing programme over time, using weeks as time units. Initially, the population database is interrogated to identify CRC cases occurring in the current week. Ascertainment and acceptance of genetic testing is determined using probability algorithms, with test results reflecting true mutation status. Another algorithm is run to determine if mutation carriers will allow their relatives to be contacted. If the outcome is affirmative, the CGTM then retrieves relatives of the newly identified mutation carrier from the population database, beginning a new cycle of ascertainment and testing.

All simulations were based on applying cascade genetic testing to a 'depth' of all first- and second-degree relatives. To reflect the long-term nature of the strategy, the CTGM simulated cascade genetic testing over a 20 -year period. Restricting genetic testing to early-onset cases is a commonly used approach to enrich for mutation carriers. For the purposes of the study, it was assumed that all CRC cases presenting under the age of 55 would be offered MMR gene mutation analysis as part of routine clinical practice. Realistic delays were also built into the model system.

Uptake of cascade genetic testing, determined by both ascertainment and acceptance rates, is likely to have a significant impact on the above outcomes. To study this, two sets of input parameters were defined:

(i) Optimum: assumes $100 \%$ ascertainment and acceptance and thus provides an indication of the theoretical utility of cascade genetic testing.

(ii) Realistic: current 'best estimates' of ascertainment and acceptance rates, based on published data $[1,20$, 21] and the practical experience of our research group. These represent conservative assumptions about what may be achievable in practice.

Table 1 provides a summary of parameters used to inform the CGTM.

Strategy for evaluating cascade genetic testing using integrated model system

The two models were considered as one integrated system. Each set of results was generated by running the GPM first, and then using the CGTM to model cascade genetic testing in the simulated population database. Variation is therefore the net result of variability in the entire model system. The number of repeat simulations was minimised to generate robust estimates whilst reducing computing time. Results presented here are based on mean estimates from multiple simulations $(\mathrm{n}=25)$ run with identical parameters. Standard deviation and confidence intervals were calculated by standard methods.
Table 1 Key parameters for Cascade Genetic Testing Model

\footnotetext{
a Eligible individuals aged less than 18 years were not processed until they reached this minimum age

b Practical experience suggests that ascertainment of relatives can be a prolonged process, and accordingly the 'realistic' set of simulations modelled presentation of relatives over a period of up to 2 years
}

\begin{tabular}{ll}
\hline Parameter type & Parameter estimate applied \\
\hline Depth of cascade genetic testing & $\begin{array}{c}\text { First- and second-degree } \\
\text { relatives } \\
\text { Length of programme }\end{array}$ \\
$\begin{array}{l}20 \text { years } \\
\text { Age limit for index cases }\end{array}$ & 55 years \\
Age limit for relatives & 75 years \\
Minimum age for genetic testing & 18 years \\
Sensitivity of genetic test & $100 \%$ \\
Specificity of genetic test & $100 \%$ \\
Realistic ascertainment rate for colorectal cancer cases & $90 \%$ \\
Realistic acceptance rate for genetic testing by colorectal cancer cases & $85 \%$ \\
Realistic proportion of index cases permitting/facilitating ascertainment & $98 \%$ \\
$\quad$ of relatives & \\
Realistic acceptance rate for genetic testing by relatives & $50 \%$ \\
Time required for genetic test in colorectal cancer case & 52 weeks \\
Time required for specific genetic test in relative of carrier & 1 week \\
Optimal time for ascertainment of relatives & $4-12$ weeks \\
Realistic time for ascertainment of relatives & $1-2$ years
\end{tabular}




\section{Results}

Cascade genetic testing identifies mutations in affected individuals, or in relatives of these index cases. Inevitably, some relatives will have developed disease previously, whereas others will be asymptomatic. This latter group constitutes the key outcome against which the effectiveness of cascade genetic testing can be judged. Figures 1 and 2 illustrate the cumulative total number of carriers identified in each category, in simulations using, respectively, 'optimal' and 'realistic' assumptions about ascertainment and acceptance. Estimates are based on the mean value from multiple simulations, with $95 \%$ confidence intervals.

Under optimum ascertainment and acceptance rates, simulations suggested that an average of $140 \quad(95 \%$ $\mathrm{CI}=135,145)$ index cases could be identified over 20 years, at a rate of approximately seven per year. The corresponding average number of asymptomatic mutation carriers identifiable through cascade genetic testing was $302(95 \% \mathrm{CI}=287,317)$. A further $104(95 \% \mathrm{CI}=98$, 110) relatives who had previously developed CRC were predicted to be identifiable.
Fig. 1 Average number of mutation carriers identified in simulations assuming 'optimal' ascertainment and acceptance rates

Fig. 2 Average number of mutation carriers identified in simulations assuming 'realistic' ascertainment and acceptance rates
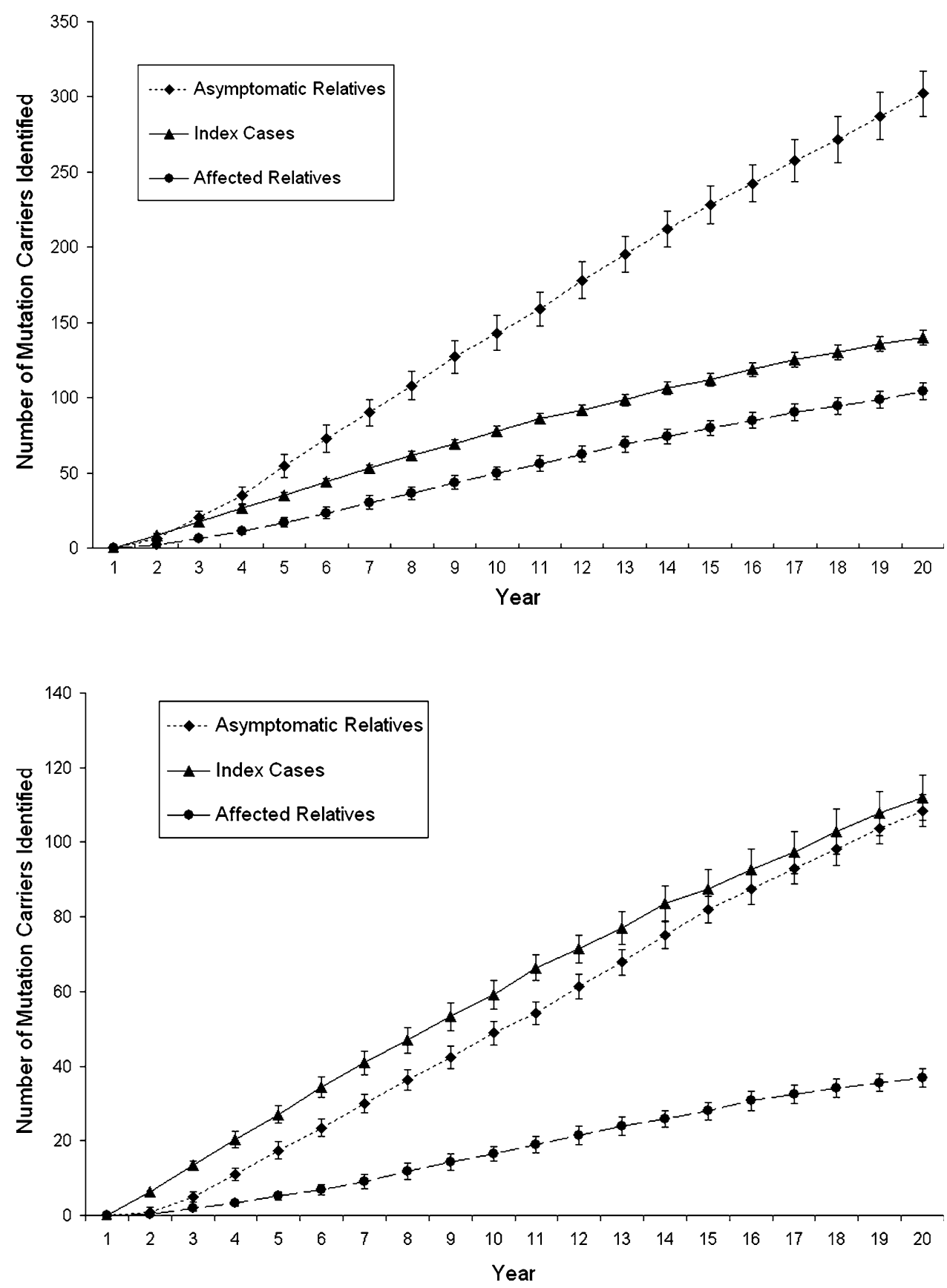
In simulated populations generated by the GPM, the average number of mutation carriers aged 15-74 years was 1,290 . This value is consistent with the published estimate used to inform the model system [9], illustrating the validity of the model in this respect. The above results suggest that under optimal (i.e. 100\%) ascertainment and acceptance rates, an average of $545(95 \% \mathrm{CI}=522,568)$ mutation carriers would be identified through cascade genetic testing over a 20 -year period. Therefore, this implies that roughly $42 \%$ of the total number of carriers in this age group aged in Scotland could theoretically be identified through a systematic cascade genetic testing programme spanning 20 years.

Results of simulations in which the CGTM was informed by more realistic estimates of ascertainment and acceptance demonstrate the major impact that these factors are likely to have on the outcomes of cascade genetic testing. These results suggest that approximately $112(95 \%$ $\mathrm{CI}=108,116)$ index cases, $108(95 \% \mathrm{CI}=102,115)$ asymptomatic relatives, and $37(95 \% \mathrm{CI}=34,39)$ previously affected relatives would be identified when realistic ascertainment and acceptance rates applied. The total number of mutation carriers that would be identified by cascade genetic testing according to these simulated results was $257(95 \% \mathrm{CI}=246,268)$, or approximately $20 \%$ of all carriers in the population. Notably, the ratio of asymptomatic carriers identified to index cases ascertained is much lower when 'realistic' ascertainment and acceptance rates for relatives are applied.

The ratio of asymptomatic carriers identified to genetic tests performed is a key indicator of the efficiency of cascade genetic testing. The relatively complex process of searching for an unspecified MMR gene mutation in a CRC case is likely to be the most resource-intensive aspect of a cascade genetic testing programme. Simulations using the CGTM suggest that under optimal ascertainment and acceptance parameters, approximately 8,400 potential index cases need to undergo genetic testing in order to identify 302 asymptomatic relatives, a ratio of about $28: 1$. In the more realistic simulations, an average of 6,500 potential index cases underwent genetic testing and around 112 asymptomatic relatives were identified, an overall ratio of about 58:1 that suggests that limitations in ascertainment and acceptance rates make the process far less efficient.

Extending the time period of cascade genetic testing beyond 20 years in these simulations showed that this strategy remains useful over an even longer period, although return diminishes slightly as the pool of untested mutation carriers dwindles. Simulations under 'optimal' assumptions suggested that about $72 \%$ of carriers could be identified after 40 years. This observation implies cascade genetic testing could in theory identify the majority of carriers in the population, but is unlikely to do so within a reasonable timeframe.

\section{Discussion}

We have developed a robust computer model system that facilitates the study of cascade genetic testing, focussing on its theoretical and practical utility in a specific clinical context.

Precise outcomes of cascade genetic testing will be population-specific due to variations in demographics and genetic epidemiology. Penetrance may vary as the specific mutations present and the proportion of hMLH1 compared to hMSH2 mutations will differ between populations. In addition, the behaviour of individuals in a cascade genetic testing programme may be influenced by social context and external factors such as the availability of free healthcare. Nonetheless, Scotland serves as a typical example of a developed country with a high incidence of CRC and low prevalence of MMR gene mutations. Hence, the methods, results and conclusions of this work have much wider relevance.

Model outcomes are critically dependent on input data, so the quality of these data merits further consideration. Robust national statistics were used to simulate the demographics of the Scottish population and to simulate CRC epidemiology, although early historical data and projected estimates had less precision. In contrast, data on family size and structure was limited and relied heavily on one publication [22]. Input data relating to the genetic epidemiology of MMR gene mutations were regarded as assumptions. Published penetrance estimates vary widely $[2,3,6]$, and ascertainment bias in previous studies may have led to overestimation [21]. This study considered all mutations in hMLH1 or hMSH2, although these are highly heterogenous and mutations in other MMR genes may also be clinically relevant. More detailed information could not be incorporated into the simulations because of the lack of robust input data.

Due to the stochastic nature of the model and the limitations outlined above, both random variation and some minor systematic differences were evident between simulated data and the 'actual' parameters of the Scottish population. Nonetheless, the system reliably produced simulated populations that were representative of the actual Scottish population in terms of demographics, genetic epidemiology, and family size and structure.

The depth to which cascade genetic testing is applied is a crucial consideration. It is desirable to test only close relatives with a very high probability of having a mutation, but this can be rather restrictive, especially in small families. Conversely, extending cascade genetic testing to 
distant relatives is more resource-intensive and may lead to reduced uptake. In reality, factors such as family history and test results in other relatives may also guide the cascade process.

In this, as in any, cascade genetic testing programme, the identification of index cases is extremely important and potentially a major limiting factor [23]. Applying genetic tests to CRC cases is the simplest approach. To improve efficiency, genetic testing can be targeted to a sub-group of cases with early onset. Family history provides an alternative means of targeting testing, although use of the stringent Amsterdam criteria [24] would miss a large proportion of carriers, and high risk families may not be representative [21]. Incorporating information on tumour pathology into the genetic testing strategy could also be an effective means of targeting testing [25]. Specific approaches, such as the two-stage model described by Barnetson et al. [26] which incorporated clinical variables in stage 1 and microsatellite instability and immunohistochemistry in stage 2, may prove useful. However, data on the use of pathology features in this context is limited, and the required pathology data are not always routinely collected. Because of this, and the inherent limitations and complexity of using family history criteria, the age alone model was chosen for the current study. Although their inclusion would theoretically permit identification of additional index cases, other cancers associated with MMR gene mutations were not simulated due to lack of quality evidence to support estimates of penetrance and test uptake.

Under optimum conditions, simulations suggested that approximately 140 index cases could be identified over 20 years. In reality though, ascertainment and acceptance will be sub-optimal [20, 27-29]. Results based on more realistic estimates, which suggest that 112 index cases could be identified, are thus more appropriate.

Simulations suggested that cascade genetic testing could theoretically identify about $40 \%$ of MMR gene mutation carriers in Scotland, most of which were predicted to be identified at an asymptomatic stage. This is consistent with estimates of theoretical effectiveness presented by Krawczak et al. [15], although results are not directly comparable since this previous study had no chronological component, and did not relate to a specific disease or population.

Published data concerning the achievable uptake for a cascade genetic testing programme is limited, and relates to large hereditary non-polyposis colorectal cancer (HNPCC) families. Figures quoted are highly variable, ranging from 33.6 [29] to $75 \%$ [27]. Low education and depression act as barriers to uptake [20], and other factors, including age, sex, and relationship to mutation carrier may also be involved [20, 27, 29]. The manner in which relatives are approached is another vital factor. Current 'best practice' in clinical genetics, designed to comply with ethical guidelines, dictates that communication with relatives must be initiated through the index case or another family member. The onus is then on the relatives to contact a genetics clinic. For the current study, this difficulty was addressed by using an estimate of $50 \%$ to reflect the overall proportion of eligible relatives who will undergo a genetic test in 'realistic' simulations. Based on the practical experience of our research group, we consider this to be a conservative estimate.

Using 'realistic' ascertainment and acceptance rates as input data for the CGTM produced results that are strikingly different to theoretical outcomes. These simulations suggest that a 20-year cascade genetic testing programme would identify a much smaller number of mutation carriers; approximately 260 , or $20 \%$ of the population total. Less than half of these carriers are likely to be identified while asymptomatic. This may be regarded as a relatively poor return from a long-term, resource-intensive testing programme, although identifying a group of mutation carriers does offer the potential to trace mutations in future generations.

The identification of asymptomatic MMR gene mutation carriers is merely the first step towards disease prevention in this population sub-group. Carriers are recommended to undergo intensive surveillance, ideally comprising biennial colonoscopy [30]. Such screening has been shown to reduce $\mathrm{CRC}$ incidence and mortality in MMR gene mutation carriers [8]. In practice though, incomplete compliance with surveillance is likely to constrain the potential health benefits [8, 29].

Our findings support the hypothesis that cascade genetic testing has the theoretical potential to be both effective and efficient as a means of identifying MMR gene mutation carriers from within a population. However, we have illustrated that incomplete ascertainment and acceptance has a significant impact. The practical barriers to pursuing cascade genetic testing effectively must therefore be given due consideration when deciding on the appropriateness of implementing this strategy.

In summary, we provide data on the potential effectiveness and efficiency of cascade genetic testing both in theory and in practice. These data and the associated observations and conclusions that have been prompted can be used to inform decisions about the use of cascade genetic testing as part of a strategy to address the health needs of MMR gene mutation carriers.

Acknowledgements This work was funded by the Chief Scientist Office of Scotland, grant no. CZH/4/145. The University of Edinburgh acted as sponsor, with vital collaboration from Heriot-Watt University. In addition to the work carried out by the authors, we acknowledge the expert input from Kenneth MacLeod regarding computer programming, and the support provided by Rosa Bisset. 


\section{Appendix 1}

Key input data to Genetic Population Model

\begin{tabular}{lllll}
\hline Input data & $\begin{array}{l}\text { Point } \\
\text { estimate } \\
\text { (if applicable) }\end{array}$ & $\begin{array}{l}\text { Source/ } \\
\text { reference }\end{array}$ & $\begin{array}{l}\text { Comments on data } \\
\text { accuracy/quality }\end{array}$ & Generalisability \\
& & & \\
\end{tabular}

Population size

$5,094,800(2005)$

GROS data

Demographic structure

Deaths (historical data)

Deaths (projections)

Births

Births (projections)

Disease incidence

Disease incidence (projections) Penetrance of MMR gene
mutations

Prevalence of MMR gene mutations

\section{GROS Robust estimate}

GROS Robust national data

GAD Projections only

GROS Robust national data

GAD Projections only

ISD

To age 70 :

males: $80 \%$

females: $40 \%$

ISD

Projections, based on current trends and demographic projections

Scientific Limited sources of data, various literature methods, wide confidence intervals
1:3,139 (95\% CI Scientific One source of data only, wide $1: 1,247,1: 7,626)$

Scotland is a relatively small country but data may be extrapolated to populations with similar demographics

Scotland has a demographic structure that is typical of an aging population in a developed country

Scotland is broadly representative of developed countries with respect to mortality rate

The projected continuation of decreasing mortality rates for Scotland is typical of developed countries

Fertility in Scotland, as with many developed countries, has decreased during the latter part of the 20th century

The projected continuation of decreasing fertility rates for Scotland is typical of developed countries

Scotland has a relatively high incidence of colorectal cancer, but incidence is comparable with other countries in which the disease is a public health issue

The increasing incidence projected for Scotland is reflected in many other populations

There is currently no evidence of population variations since differences in published estimates are likely to be due to methods used and lack of statistical power. However, penetrance may vary according to the precise mutation involved and numerous other factors, and may therefore vary by population

Prevalence data are scarce, and the single published estimate relates to Scotland. Prevalence is likely to vary by country, particularly when common founder mutations are present in the population (e.g. Finland), but there is no evidence to suggest that Scotland is not representative in this respect 
Appendix 1 continued

\begin{tabular}{|c|c|c|c|c|}
\hline Input data & $\begin{array}{l}\text { Point } \\
\text { estimate } \\
\text { (if applicable) }\end{array}$ & Source/reference & $\begin{array}{l}\text { Comments on data } \\
\text { accuracy/quality }\end{array}$ & Generalisability \\
\hline Disease survival & & ISD & Robust national data & $\begin{array}{l}\text { In terms of survival, Scotland is } \\
\text { representative of countries with } \\
\text { modern health care systems }\end{array}$ \\
\hline Family structure & & ONS & $\begin{array}{l}\text { Data on frequency distribution of } \\
\text { children as well as completed } \\
\text { family size is limited to one } \\
\text { reliable source }\end{array}$ & $\begin{array}{l}\text { Family structure may vary across } \\
\text { populations according to } \\
\text { cultural, social and demographic } \\
\text { trends. However, Scotland has a } \\
\text { stable population which is } \\
\text { useful for comparisons, and the } \\
\text { observed trend of decreasing } \\
\text { family size leading to stabilizing } \\
\text { or decreasing population is } \\
\text { common }\end{array}$ \\
\hline
\end{tabular}

GROS = General Register Office Scotland, GAD = Government Actuary's Department, ISD = Information Services Division Scotland, ONS $=$ Office of National Statistics, $\mathrm{CI}=$ Confidence interval

\section{References}

1. Aaltonen LA, Salovaara R, Kristo P et al (1998) Incidence of hereditary nonpolyposis colorectal cancer and the feasibility of molecular screening for the disease. $\mathrm{N}$ Engl J Med 338: 1481-1487

2. Aarnio M, Sankila R, Pukkala E et al (1999) Cancer risk in mutation carriers of DNA-mismatch-repair genes. Int $\mathrm{J}$ Cancer 81:214-218

3. Dunlop MG, Farrington SM, Carothers AD et al (1997) Cancer risk associated with germline DNA mismatch repair gene mutations. Hum Mol Genet 6:105-110

4. Liu B, Farrington SM, Petersen GM et al (1995) Genetic instability occurs in the majority of young patients with colorectal cancer. Nat Med 1:348-352

5. Lynch HT, de la Chapelle A (1999) Genetic susceptibility to nonpolyposis colorectal cancer. J Med Genet 36:801-818

6. Vasen HF, Wijnen JT, Menko FH et al (1996) Cancer risk in families with hereditary nonpolyposis colorectal cancer diagnosed by mutation analysis. Gastroenterology 110:1020-1027

7. Mitchell RJ, Farrington SM, Dunlop MG et al (2002) Mismatch repair genes hMLH1 and hMSH2 and colorectal cancer: a HuGE review. Am J Epidemiol 156:885-902

8. Jarvinen HJ, Aarnio M, Mustonen H et al (2000) Controlled 15year trial on screening for colorectal cancer in families with hereditary nonpolyposis colorectal cancer. Gastroenterology 118:829-834

9. Dunlop MG, Farrington SM, Nicholl I et al (2000) Population carrier frequency of hMSH2 and hMLH1 mutations. Br J Cancer 83:1643-1645

10. Brown ML, Kessler LG (1996) Use of gene tests to detect hereditary predisposition to cancer: what do we know about cost effectiveness? Int J Cancer 69:55-57

11. Coughlin SS, Miller DS (1999) Public health perspectives on testing for colorectal cancer susceptibility genes. Am J Prev Med 16:99-104

12. Ponder BA (1999) Costs, benefits and limitations of genetic testing for cancer risk. Br J Cancer 80 suppl 1:46-50

13. Terdiman JP, Conrad PG, Sleisenger MH (1999) Genetic testing in hereditary colorectal cancer: indications and procedures. Am J Gastroenterol 94:2344-2356
14. Welch HG, Burke W (1998) Uncertainties in genetic testing for chronic disease. JAMA 280:1525-1527

15. Krawczak M, Cooper DN, Schmidtke J (2001) Estimating the efficacy and efficiency of cascade genetic screening. Am J Hum Genet 69:361-370

16. Umans-Eckenhausen MA, Defesche JC, Sijbrands EJ et al (2001) Review of first 5 years of screening for familial hypercholesterolaemia in the Netherlands. Lancet 357:165-168

17. Bhatnagar D, Morgan J, Siddiq S et al (2000) Outcome of case finding among relatives of patients with known heterozygous familial hypercholesterolaemia. BMJ 321:1497-1500

18. Marks D, Wonderling D, Thorogood M et al (2002) Cost effectiveness analysis of different approaches of screening for familial hypercholesterolaemia. BMJ 324:1303-1307

19. Hadfield GS, Humphries SE (2007) Familial hypercholesterolaemia: cascade testing is tried and tested and cost effective. BMJ 335:683

20. Lerman C, Hughes C, Trock BJ et al (1999) Genetic testing in families with hereditary nonpolyposis colon cancer. JAMA 281:1618-1622

21. Quehenberger F, Vasen HF, van Houwelingen HC (2005) Risk of colorectal and endometrial cancer for carriers of mutations of the hMLH1 and hMSH2 gene: correction for ascertainment. J Med Genet 42:491-496

22. Chamberlain J, Smallwood S (2004) Estimates of true birth order for Scotland, 1945-1999. Popul Trends 117:27-42

23. Morris JK (2004) Is cascade testing a sensible method of population screening? J Med Screen 11:57-58

24. Vasen HF, Mecklin JP, Khan PM et al (1991) The International Collaborative Group on hereditary non-polyposis colorectal cancer [ICG-HNPCC]. Dis Colon Rectum 34:424-425

25. Jenkins MA, Hayashi S, O'Shea A-M et al (2007) Pathology features in Bethesda guidelines predict microsatellite instability: a population-based study. Gastroenterology 133:48-56

26. Barnetson RA, Tenesa A, Farrington SM et al (2006) Identification and survival of carriers of mutations in DNA mismatch-repair genes in colon cancer. $\mathrm{N}$ Engl $\mathrm{J}$ Med 354:2751-2763

27. Aktan-Collan K, Mecklin JP, Jarvinen H et al (2000) Predictive genetic testing for hereditary non-polyposis colorectal cancer: uptake and long-term satisfaction. Int J Cancer 89:44-50 
28. Aktan-Collan K, Mecklin JP, de la Chapelle A, Peltomaki P, Uutela A, Kaariainen H (2000) Evaluation of a counselling protocol for predictive genetic testing for hereditary non-polyposis colorectal cancer. J Med Genet 37:108-113

29. Ponz de Leon M, Benatti P, Di Gregorio C et al (2004) Genetic testing among high-risk individuals in families with hereditary nonpolyposis colorectal cancer. Br J Cancer 90:882-887
30. Dunlop MG (2002) Guidance on gastrointestinal surveillance for hereditary non-polyposis colorectal cancer, familial adenomatous polypolis, juvenile polyposis, and Peutz-Jeghers syndrome. Gut 51 suppl 5:V21-V27 